Archives de sciences sociales des religions

111 | juillet-septembre 2000

Varia

\title{
Les thánh thân dans la migration
}

Même les esprits s'acculturent

Martine Wadbled

\section{CpenEdition}

Journals

Édition électronique

URL : http://journals.openedition.org/assr/20234

DOI : $10.4000 /$ assr.20234

ISSN : $1777-5825$

Éditeur

Éditions de l'EHESS

Édition imprimée

Date de publication : 1 septembre 2000

Pagination : 149-177

ISBN : 2-222-96695-7

ISSN : 0335-5985

Référence électronique

Martine Wadbled, «Les thánh thân dans la migration », Archives de sciences sociales des religions [En ligne], 111 | juillet-septembre 2000, mis en ligne le 19 août 2009, consulté le 19 avril 2019. URL : http:// journals.openedition.org/assr/20234; DOI : 10.4000/assr.20234

Ce document a été généré automatiquement le 19 avril 2019

(c) Archives de sciences sociales des religions 


\title{
Les thánh thân ${ }^{1}$ dans la migration
}

\author{
Même les esprits s'acculturent
}

\author{
Martine Wadbled
}

1 Parmi différentes pratiques religieuses viêtnamiennes, impliquant la possession, le culte des Thánh Mẫu ${ }^{2}$, populaire dans le Nord du pays, est actuellement pratiqué en France. Pratiqué essentiellement par des femmes d'origine viêtnamienne, rapatriées à la fin de la guerre d'Indochine, en 1954, il a fait l'objet d'une étude ethnologique dans les années soixante ${ }^{3}$ et les chercheurs pensaient être les témoins des derniers moments de cette pratique, n'en imaginant pas la reproduction possible hors de son contexte d'origine. Elle ne concernait, en France, qu'une population très restreinte, et l'existence du culte semblait alors subordonnée à la durée de vie des pratiquantes.

Bien que toujours très marginale, cette pratique religieuse non seulement perdure, une « relève » timide se manifestant parmi les descendantes des rapatriées d'Indochine, mais a bénéficié d'un certain regain avec l'arrivée, à partir de 1975, d'adeptes, hommes et femmes, médiums confirmés et futurs pratiquants parmi les réfugiés viêtnamiens. Le culte est pratiqué ici ou là de façon isolée, dans le cadre familial ou privé, mais l'on assiste aussi à des regroupements plus formalisés sur le mode associatif, dans des temples spécifiques, réunissant parfois des fidèles installés en France depuis plus de quarante ans et d'autres nouvellement arrivés. Ce regain par une nouvelle population se combine à un nouveau souffle, venant du Viêt Nam, provoqué par la réouverture du pays et l'affichage d'une politique de libéralisation dont le renouveau du culte au Viêt Nam est une conséquence. Le gouvernement communiste avait totalement interdit les pratiques de ce type, qualifiées de superstitieuses, mais devant l'impossibilité de leur complète élimination et le souci d'une image de libéralisation, les tolère quoique de façon restreinte et sous son contrôle, depuis le début des années $1990^{4}$. Ainsi, les fidèles établis en France sont-ils de plus en plus nombreux à faire le voyage et profitent de l'occasion pour pratiquer le culte sur son lieu de «naissance». Si, pour l'instant, les liens renoués avec le pays n'ont pas un fort impact sur les pratiques en France, on remarque déjà quelques éléments du rituel influencés par ces nouveaux échanges, aspect dont il faudra certainement tenir compte dans les analyses à venir. 
$3 \quad$ Les sujets de réflexion que suscite ce culte ne manquent pas; entre autres, les questions de la transmission, des relations entre adeptes de France et du Viêt Nam ou d'autres pays occidentaux, de même que la pratique actuelle au Viêt Nam. Elles sont à replacer dans une recherche globale, chacune de ces questions méritant un développement spécifique. Je privilégierai ici la pratique en France.

4 La question centrale de la relation entre le social et le religieux est envisagée dans une situation particulière : la migration. Cela étant, quelle est la place et la signification de ce culte issu d'une autre tradition culturelle et religieuse dans ce nouveau contexte social et culturel français? Dans une société de culture non seulement différente mais dominante? Le contexte migratoire français inscrit de facto le fait étudié dans une situation sociologiquement minoritaire. Ainsi, l'insistance sur la migration met en lumière les modalités de rencontre des deux univers culturels en présence et inscrit une analyse dans la problématique générale de l'acculturation telle que l'a définie Roger Bastide. Nous tenterons d'apporter des éléments de réponses à ces interrogations en abordant ici les permanences, les éventuelles transformations des pratiques en France, en les comparant avec celles observée au Viêt Nam, et essayant de mettre en relief des enjeux du culte au Viêt Nam et en France. Ainsi, après une rapide présentation de l'univers spirituel viêtnamien et du culte des Thánh Mẫu ${ }^{5}$, on montrera dans une première partie, les relations sociales en œuvre dans le culte et dans une seconde, la part dans la construction identitaire des personnes d'origine viêtnamienne en France.

\section{Thần et Thánh dans l'univers spirituel viêtnamien}

5 Bien que fortement sinisé, le cadre général de croyance viêtnamien n'en est pas moins original, conjuguant un animisme très ancien, des croyances taoïstes, la doctrine confucéenne et le bouddhisme du Mahayana. Davantage une juxtaposition de diverses croyances que le syncrétisme que l'on évoque souvent, l'univers spirituel viêtnamien est d'abord marqué par ce fonds religieux commun à de nombreux groupes ethniques d'Asie du Sud-Est qu'est la religion des esprits.

6 Comme dans de nombreuses sociétés, la conception viêtnamienne du monde est fondée sur l'interdépendance de deux univers, l'un visible a priori, univers des humains, d'ici-bas, et l'autre non immédiatement perceptible, un univers peuplé d'entités invisibles la plupart du temps et de différentes natures : esprits liés aux forces de la nature, héros historiques divinisés, esprits gardiens, protecteurs sous différentes formes, (animales ou anthropomorphes), âmes humaines honorées comme ancêtres ou errantes, démons... Bonnes ou mauvaises, les entités sont toujours dotées de pouvoirs considérés comme efficients par les humains qui en invoquent certaines pour protéger leurs entreprises terrestres, garantir leur santé, leur bien être. En échange de cette protection, ou pour éviter les tourments des esprits mauvais, les humains doivent, sous forme de pratiques rituelles, faire preuve de reconnaissance, de respect, de soumission.

7 La terminologie employée pour désigner les entités suivant la hiérarchie de puissance et leur nature (bonne ou mauvaise) est complexe et nous nous limiterons à la désignation de celles du cadre religieux qui nous intéresse. Toutes les entités du culte des Thánh Mẫu sont désignées comme: thánh ou parfois thánh thần. Cette dénomination est une juxtaposition de deux termes : thần ${ }^{6}$, appellation générique évoquant l'idée de magique, merveilleux, miraculeux même, appliquée à des entités d'essences différentes. Elles sont, 
soit de nature divine, soit des quintessences de la nature, ou même des esprits humains divinisés. Nanties d'une puissance, bienfaisante en principe, elles peuvent se révéler malfaisantes en certaines circonstances. Le mot thánh, renvoie plutôt à l'idée de vénérable, pur, et devait semble-t-il être réservé autrefois aux entités les plus élevées, bouddhiques notamment. Aujourd'hui ce terme s'applique systématiquement, aux thần les plus élevés comme : les Thánh Mẫu, ou le héros national divinisé (le général Trần Hưng Đao) comme aux autres entités moins élevées dans la hiérarchie du panthéon. De façon courante, les adeptes ont pris l'habitude d'employer le seul terme thánh pour toutes les entités du panthéon et désignent comme thần des entités de moindre puissance, («que l'on craint moins » dit un médium) protectrices des villages, des terrains, des temples et des pagodes, n'appartenant pas au panthéon. Ce changement d'appellation est interprétable de deux façons, selon l'angle choisi. Pour les adeptes, il s'agit d'un simple glissement de langage n'ayant pas entraîné de modifications dans l'appréciation, des natures des entités et de la hiérarchie de la vénération. Pour ma part, j'y vois plutôt l'empreinte d'une certaine bouddhicisation du culte. Lorsque l'on évoque les Thánh dans les conversations, les médiums préfèrent, à l'appellation générique, une identification personnelle, simplifiée la plupart du temps et basée sur la place du Thánh dans la hiérarchie du panthéon. Ainsi, on évoquera l'incarnation de Quan Lờn đệnhất (le premier grand mandarin), de Ông Hoàng Mười (monsieur le dixième prince), de Cô Chín (neuvième demoiselle). Certains, peu nombreux il est vrai, estiment pourtant que ces termes d'adresse ne marquent pas assez la déférence et privilégient des expressions comme : " Vưõng Mẫu » ou "Chu vị thiên gía » inspirées de formules employées autrefois à l'intention des personnalités de la cour impériale.

8 La terminologie française du culte et des adeptes varie selon les points de vue. Les ethnologues, sociologues ont utilisé, jusqu'à présent, des expressions en référence aux adeptes : culte des «médiums", culte des " đồng ",ou des «bà đồng "; terme utilisé pour désigner les médiums femmes et qui signifie littéralement: "dame servante ${ }^{7}$ ». En référence à l'organisation du panthéon, le culte est parfois dit « des génies des Quatre Palais » ou est encore qualifié à partir d'expressions employées par les bà đồng : «lên đồng » (monter le serviteur) pour rendre compte de la modalité caractéristique de la possession ou « hầu bóng " (servir les ombres) qui définit la situation des médiums. Pour ma part, je reprendrai la terminologie employée actuellement par la plupart des adeptes rencontrés. Plutôt que thần qui est pourtant l'appellation générique adéquate, j'utiliserai de préférence le terme thánh. Toutefois, le mot français « esprit » quoique moins précis me semble également opportun; les thánh sont inclus dans l'univers des esprits au sens large, opposé au monde des humains. Pour les médiums et le culte, je reprendrai également de préférence les expressions des adeptes : bà tồng pour les médiums femmes, ông đồng pour les médiums masculins et Thánh Mẫu pour le culte.

\section{Le panthéon des Thánh Mẫu}

Comme il a déjà été dit plus haut, ce culte, populaire au nord du Viêt Nam est une pratique médiumnique parmi d'autres répandues dans le pays. Dans le centre, à Huê, on rencontre une pratique très proche. Le panthéon est composé, en majorité, des mêmes entités mais l'on observe des variations notables dans le déroulement du rituel. Le Sud est concerné par ce culte dans une moindre mesure et c'est surtout la fuite au Sud-Viêt Nam d'une partie de la population du Nord, à la fin de la guerre française en Indochine, en 1954, qui l'a introduit dans cette partie du pays. 
10 De façon schématique, le panthéon comprend plusieurs niveaux d'entités suivant une hiérarchie décroissante de la vénération. ve et reproduit, en partie, l'organisation sociale terrestre de l'époque de la mise en place du culte sous sa forme actuelle, que l'on situe généralement aux XVIII ${ }^{e}$-XIX ${ }^{e}$ siècles'. La position des thánh dépend d'un ordre hiérarchique déterminé par le rang, le sexe, l'âge. Ils exercent ainsi des fonctions différentes selon qu'ils sont mandarins, dames, princes, demoiselles ou jeunes princes enfants, du palais de l'eau, du ciel, etc... En principe ce sont des protecteurs, bienfaisants, guérisseurs mais dont la colère est à craindre lorsqu'ils jugent n'avoir pas été traités avec les égards qui leur sont dus. Leurs personnalités sont bien marquées et connues. Certains sont rieurs, d'autres mélancoliques, sérieux, d'autres encore capricieux, font des effets de coquetterie. Placés au sommet de la hiérarchie, ils sont plus distants, réservés, autoritaires, leurs apparitions sont plutôt courtes, et plus on «descend» dans la hiérarchie, plus le contact avec les humains est convivial, prolongé.

17 La structure de base du panthéon est stable, mais, au Viêt Nam, on observe des variations selon les régions. Il comprend un nombre de thánh qu'il est difficile de quantifier précisément, mais que l'on organise souvent comme suit ${ }^{10}$ :

18 - Les trois Thánh Mẫu : la Mère céleste (Thánh Mẫu Thượng Thiên), la Mère des Hautes Régions des monts et forêts (Thánh Mẫu Thượng Ngàn) et la Mère des Eaux (Thánh Mẫu Thủy Phủ). Elles dominent à un niveau supérieur. Entités primordiales, elles ne s'incarnent jamais véritablement lors des cérémonies.

19 - dix grands mandarins (Quan Lớn);

20 - douze dames (Châù);

21 - dix princes (Ông Hoàng) ;

22 - douze demoiselles (Cô) ;

23 - dix ou douze petits princes enfants (Cậu). 

insèrent le culte dans le cadre bouddhique. Toute cérémonie commence et prend fin par
des prières à Bouddha et l'on emploie la formule rituelle bouddhiste « A Di Đà Phật » pour
louer, remercier les thánh pendant les incarnations.

Prise de possession sous le voile.

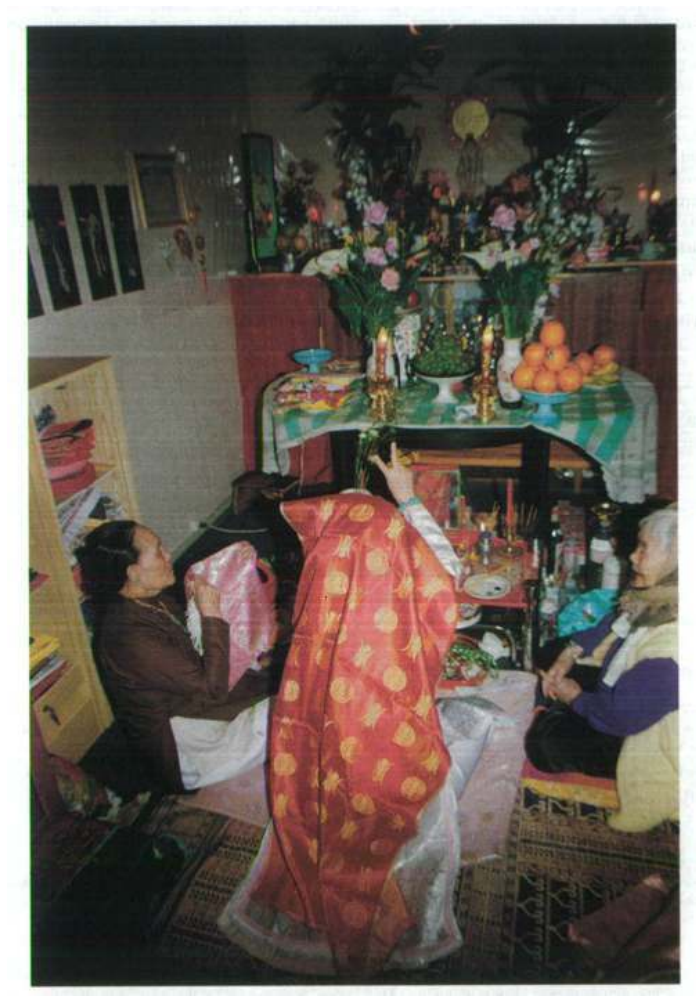

Le medium indique par un geste de la main l'identité du Thánh (ici : la deuxième Dame). Autel privé, Rennes, 1993.

Le cadre cérémoniel est soigneusement préparé, en fonction d'un calendrier religieux et les possessions sont généralement « heureuses ${ }^{11}$ ", se déroulant dans une ambiance plutôt détendue ${ }^{12}$. En règle générale, la cérémonie est le fait d'un seul médium, mais il arrive que parmi les personnes de l'assistance, des bà đồng confirmées la plupart du temps, certaines soient possédées spontanément par un thánh, et elles se joignent alors à l'officiante principale le temps de l'incarnation de ce thánh. Cela se produit surtout au moment des incarnations des Cô, et deux ou trois Cô différentes peuvent s'incarner en même temps ${ }^{13}$.

Les incarnations se succèdent en respectant la hiérarchie, des plus hauts placés, les grands mandarins, aux jeunes enfants. Tous cependant ne "descendent» pas sur le médium, certains sont très fréquents, d'autres très rares Au cours d'une cérémonie, le médium incarne successivement plusieurs thánh, allant d'une dizaine à une vingtaine 
d'incarnations différentes selon les personnes. Les incarnations, s'effectuent en séquences dont les phases principales se répètent. ${ }^{14}$ La durée d'une cérémonie n'est pas strictement définie. De façon générale, elle varie entre quatre et sept heures environ, selon le nombre et la durée des incarnations effectuées par la bà đồng.

Incarnation d'une « Demoiselle des Montagnes».

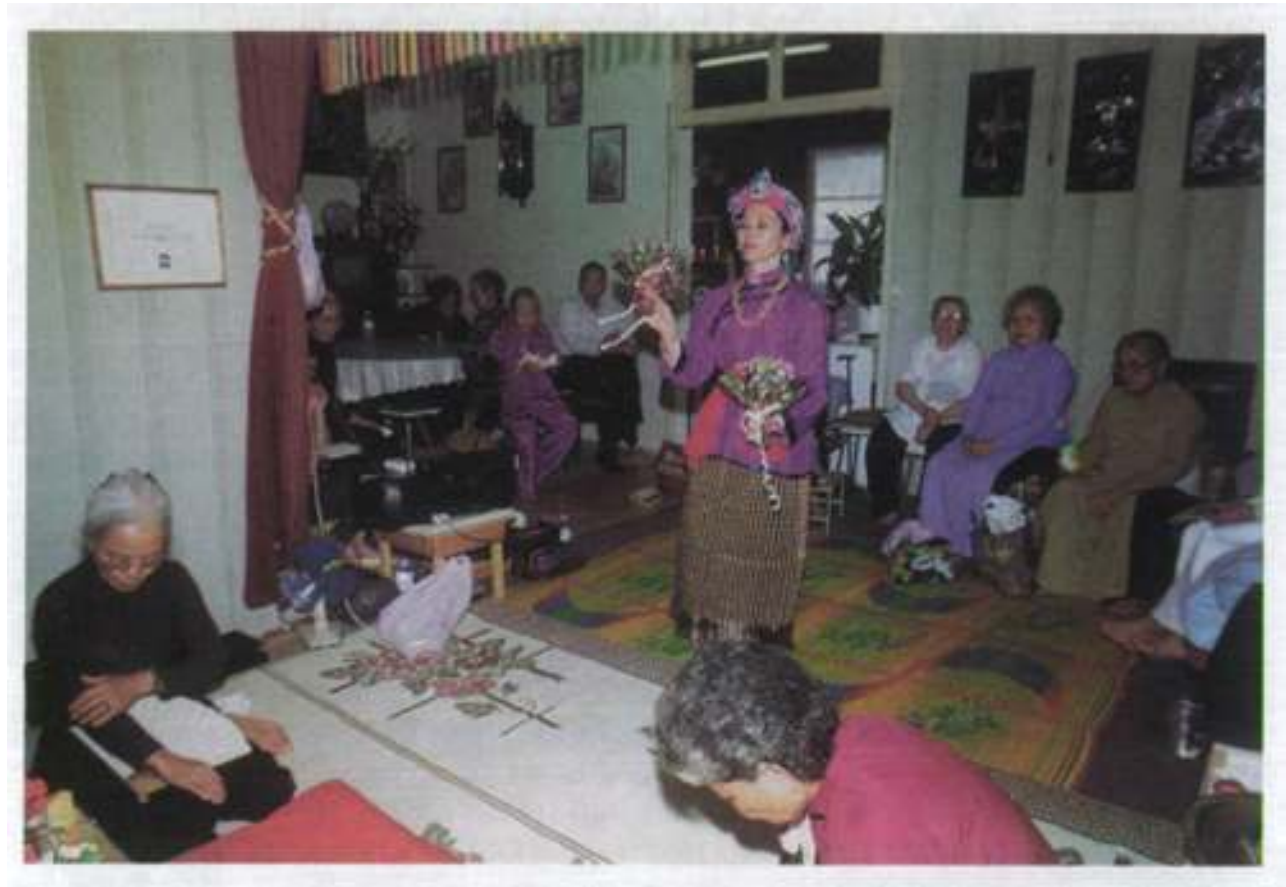

Lot-et-Garonne, Autel privé, 1995

\section{Le culte : une forme de relation sociale}

31 Même s'il ne concerne qu'une partie de la population au Viêt Nam et un groupe encore plus restreint en France, le culte instaure une relation sociale collective, avec ses règles, ses contraintes ses enjeux matériels, relationnels. Pour la saisir et la comprendre, nous allons situer la possession, décrire les relations entre les différents protagonistes: les médiums et le public des invités aux cérémonies et nous essaierons de dégager les fonctions de ce culte. Les situations choisies pour illustrer les relations, et les différentes fonctions exercées par la bà đồng, au cours d'une séance de possession, sont extraites de l'observation en France. Si les exemples relèvent du cadre français, le fonds reste cependant commun aux deux pays. Nous verrons dans une autre partie, la spécificité du contexte français et ses conséquences.

\section{1 - Le rapport bà đồng /thánh dans la possession}

La possession est d'emblée perçue comme la domination d'un esprit par un autre, plus fort. Les termes qui caractérisent la situation sont révélateurs du rapport de domination qui s'établit entre le médium et l'esprit. En viêtnamien, on dit de l'esprit qu'il « descend » (giáng) sur le médium, le "monte» telle une monture (lên), qu'il l'a "choisi» (chấm), "attrapé » (bắt) pour en faire son "serviteur » (đồng $\left.{ }^{15}\right)$. Le médium est «le siège » (ghế đệm) des «ombres » (bóng) qu'il «sert » (hầu), dont il est le soldat (lính) pendant tout le 
temps de l'incarnation de chacun (gián $\left.{ }^{16}\right)$. Différents cultes de possession existent au Viêt Nam, pour lesquels il est nécessaire de faire la distinction entre les pratiques masculines et féminines. Les hommes sont le plus souvent attachés à un esprit, un thánh particulier, tel le général Trần Hýng Đạo,dont le culte comporte des aspects parfois violents alors que les médiums au service des thánh des Quatre Palais sont en très grande majorité des femmes (plus de $90 \%$ ). Bien que numériquement minoritaires, les hommes ne sont pourtant pas rares. Mais la connotation féminine du culte est prépondérante car ces adeptes masculins sont souvent décrits comme efféminés, à tendance homosexuelle. Notons cependant que lorsque les hommes sont présents, en France comme au Viêt Nam, ils occupent une place privilégiée dans l'organisation du culte. Souvent responsables de temples, ils dirigent les cérémonies. ${ }^{17}$

\section{2 - L'interdépendance bà đồng/invités}

Les invités, sont au cœur d'une double réciprocité avec, d'une part le rapport bà đồng/ invités et d'autre part la relation invités/thánh. La relation bà đồng/public est construite en partie par la bà đồng qui lance des invitations individuelles, l'accès aux cérémonies étant, en principe, réservé aux invités : médiums et fidèles (« appelées » non encore initiées, ou simples fidèles), mais aussi amis. Cette sélection du public est à comprendre non comme une volonté de fermeture du culte aux regards extérieurs, une sorte de mise au secret, mais plutôt comme une manière de maîtriser la situation, de mettre les atouts de son côté pour la cérémonie, en s'assurant le concours d'un public a priori favorable. La composition du public est cependant fluctuante. L'effectif varie selon les cérémonies, selon les moments également car si certaines personnes disponibles assistent à toute la cérémonie, d'autres arrivent en cours ou partent avant la fin. Un point important motive des invités à se déplacer: la réputation de la bà đồng. Certaines personnes sont réputées plus «fortes » que d'autres, plus réceptives aux esprits. Leurs possessions plus spectaculaires que la moyenne sont perçues d'autant plus chargées des bienfaits des thánh, et ce sont elles qui, généralement, ont un pouvoir de guérison. Ainsi, les invités, nombreux à répondre à l'invitation de telle bà dồng, seront moins enclins à se déplacer pour une médium de moindre renom. Il s'établit donc une relation d'interdépendance entre la bà đồng et les invités. Ceux-ci, sont honorés d'avoir été choisis, et celle-là doit à l'assistance présente l'entretien de sa renommée. Les invités sont, en outre, les indispensables témoins de la manifestation des esprits.

Les thánh sont des personnages puissants, que l'on doit respecter et pour certains, cette puissance est assortie d'un statut social élevé, pris sur le modèle impérial. On comprendra aisément qu'ils aient besoin d'une assemblée qui les honore, une sorte de cour qui les loue, les félicite de leur prestance, les admire. Chacun des esprits (à l'exception des plus élevés dans la hiérarchie) dispense alors ses bienfaits, considérés comme autant de bonheurs, de fortunes (lọc), à la servante mais aussi aux personnes de l'assistance. Les fruits, légumes, gâteaux, petits objets, pièces de monnaie ou billets qu'a préparés la bà đồng en offrande sont les cadeaux qu'offrent les thánh à l'assemblée. Chacun bénéficie alors des bienfaits, de la protection des thânh: les personnes les plus proches du culte servies les premières, recevront les meilleures "parts", les spectateurs les moins impliqués recevront en second lieu des cadeaux un peu plus modestes. Les premiers sont bien sûr honorés de cette faveur mais l'effet bénéfique est néanmoins dans tous les présents faits par les thánh. Les cadeaux, dans l'ensemble, ont peu de valeur marchande, c'est la force bénéfique dont ils sont chargés qui compte. 


\section{3 - Les fonctions du culte}

Si l'autothérapie vaut pour toutes les adeptes, la fonction de guérison est réservé à un nombre restreint de personnes. Quelques bà đồng seulement, réputées nanties d'un « căn » (principe vital) particulièrement fort, ont la capacité de guérir, et cela uniquement lorsqu'elles sont possédées par certains thánh reconnus guérisseurs telle " Cô bõ " (troisième demoiselle du palais des Eaux). L'eau et l'encens jouent là un rôle prépondérant ; l'eau en tant qu'élément de ce génie et principe de vie, et l'encens comme vecteur de communication entre les humains et les entités spirituelles. L'invocation d'un 
thánh guérisseur peut se faire à titre curatif ou préventif. Ainsi, concrètement, à titre curatif, lorsque la troisième demoiselle s'incarne, la personne souffrante lui demande (la plupart du temps par l'intermédiaire d'une assistante) de bien vouloir la guérir de telle ou telle affection. Le génie, après avoir aspiré un peu de fumée d'encens, à l'extrémité de baguettes, la recrache dans une tasse ou une bouteille d'eau minérale et la donne à boire à la personne malade. Passée dans la bouche du médium, la fumée d'encens est " chargée » de la force bénéfique du génie, une force qui se propage à l'eau dans laquelle est crachée la fumée. La posologie varie selon les cas: une petite tasse à boire sur place ou une bouteille entière à emporter chez soi et à consommer en quelques jours. L'encens seul suffit parfois à soigner; dans ce cas, la bà đồng dessine des signes dans l'air, à l'aide d'une baguette d'encens allumée, au-dessus de la partie malade. De façon préventive, le génie recourt à l'une ou à ces deux mêmes méthodes. On peut prendre ici l'exemple de la fille d'une bà đồng qui était régulièrement protégée de ces deux manières, les signes étant esquissés au-dessus de la tête de la jeune fille, une étudiante de troisième cycle universitaire, et dont le bon état de la tête, siège des capacités intellectuelles, était jugé comme particulièrement important. De plus, elle recevait une tasse d'eau à boire. Une autre méthode préventive consiste à recevoir du génie, de la fumée d'encens sur les mains et à se passer les mains sur le visage pour bénéficier de la protection. Cette action est généralement dispensée généreusement, sans demande particulière, aux personnes de l'assemblée.

Le pouvoir de guérison est un des éléments qui constituent la notoriété d'une bà đồng. Si presque toutes s'essaient à l'exercice de la guérison, elles sont en fait peu nombreuses à voir leur pouvoir reconnu sachant qu'il ne leur est pas personnel, mais toujours délégué par le génie, rappelons-le.

\section{d-La divination}

40 Les personnes de l'assemblée viennent consulter un thánh particulier lorsqu'elles ont besoin de conseils pour une entreprise, ou quand une prise de décision semble difficile. Faut-il vendre, acheter un restaurant, une maison? Lors de son incarnation, il apportera une réponse par divination à l'aide de deux pièces de monnaie dont l'une des faces est peinte en noir. Les pièces sont jetées en l'air et, selon la configuration, la réponse sera positive ou négative. Ainsi, la meilleure configuration est celle d'une face noire et d'une face blanche, dès le premier lancer. Si les pièces présentent la même face, blanche ou noire, la situation n'est pas de bon augure. Il arrive fréquemment que la bà đồng, relance les pièces jusqu'à l'obtention de la bonne configuration, mais dans ce cas, si l'entreprise réussit, il y a lieu de croire que cela ne se fera pas sans difficultés. Là encore, on observe une variation de pratique entre les médiums. Très peu ont recours à la divination sous possession au cours de la cérémonie. Généralement, les pièces sont utilisées, hors la possession, avant la cérémonie, pour savoir si celle-ci est placée sous de bons auspices.

\section{$e$ - Conseils ou intercession auprès des instances supérieures}

41 Le thánh peut avoir un rôle de conseiller direct ou intercéder auprès d'une entité supérieure qui ne s'incarne pas. Prenons l'exemple de cette femme, une Eurasienne en France depuis la fin de la guerre d'Indochine, dont la vie était difficile. Accident de voiture, vision pessimiste de la vie, tout paraissait aller de travers, et ce depuis la mort de son chien malade, dont elle avait décidé l'euthanasie. Le dixième prince la sermonna 
vertement pour avoir décidé de la mort de l'animal, dont «l'heure n'était pas encore venue » dit-il ${ }^{18}$. Le thánh lui expliqua qu'elle devait tous ses problèmes au fantôme de son chien qui la tourmentait pour avoir hâté sa fin. Il donna à la femme en larmes, très émue, quelques offrandes alimentaires et des baguettes d'encens à déposer à l'emplacement habituel du chien, (un fauteuil en l'occurrence) pour apaiser l'âme de l'animal. Lui recommandant de ne plus agir de cette façon, il lui assura une amélioration prochaine de sa vie. Une autre fois, il conseilla une femme hésitant sur une question d'investissement financier professionnel. Le thánh peut également protéger sans qu'une requête ait été déposée, en s'adressant directement à la personne concernée dans l'assistance. Ainsi, quelque temps avant un séjour au Viêt Nam, au cours d'une cérémonie, la dame des eaux m'octroya une protection particulière, en traçant devant moi des signes avec la fumée d'encens, et me recommanda d'aller visiter un temple bien précis qui lui est dédié au Viêt Nam.

Lorsqu'on examine les différentes fonctions, on s'aperçoit que certaines comportent deux aspects, l'un manifeste et l'autre latent. La fonction d'autothérapie en est un exemple. D'une part la bà đồng est« guérie », c'est l'aspect manifeste, et d'autre part, cette guérison est conditionnée à la pratique, dans la crainte de voir resurgir des difficultés. La fonction latente est donc d'assurer le recrutement du culte. Dans les faits, le culte est rarement présenté par les médiums sous ce jour contraignant; au contraire, les avantages seront mis en avant, et la possession d'après les adeptes, est vécue de façon sereine.

Cependant quelques femmes, parmi les plus âgées, ont cessé la pratique. Mais il leur a fallu demander aux thánh la permission de se retirer du service. Elles invoquent leur grand âge et le plus souvent, se mettent sous la protection de Bouddha, entité jugée supérieure ${ }^{19}$. Le bouddhisme orthodoxe étant perçu comme une voie plus pure, elles délaissent l'autel des thánh pour celui dédié aux seules divinités bouddhiques. Les thánh, toujours intégrés à leurs croyances, sont cependant relégués à un niveau religieux inférieur. Moins assidues qu'avant la cessation, ces femmes ne dédaignent pourtant pas de se rendre aux cérémonies de bà đồng amies, lorsqu'elles sont invitées.

Le rôle de conseiller met en évidence un pouvoir sur le social légitimé par le religieux. Les thánh interviennent quelquefois dans les affaires terrestres, influant sur les décisions des demandeurs par leurs avis favorables ou défavorables. On ne peut nier l'existence, par leur truchement, d'un certain pouvoir de la bà đồng. Le travail de terrain mené jusqu'à présent n'a pas encore approfondi cette intéressante question qui nécessite une approche d'une réelle finesse, car il n'est pas toujours bienvenu de s'immiscer dans les affaires traitées entre les thánh et les demandeurs. Ces questions étant abordées sur un mode confidentiel entre les requérants et le thánh, la curiosité du chercheur est mise à rude épreuve.

On peut faire le lien entre les effets du culte dans la vie quotidienne et les interdictions dont il a fait l'objet à plusieurs reprises dans l'histoire du Viêt Nam, tant sous la monarchie que sous l'actuel régime communiste. En conformité avec l'idéologie communiste au pouvoir, l'argument officiel de la dernière interdiction était la lutte contre les superstitions. Celles-ci étaient jugées néfastes, amenant certains médiums à se ruiner dans les dépenses du rituel ou au contraire, profitant de la crédulité de la population et de leur ascendant, tendaient à leur donner un rôle influent. Les médiums ont déjà ainsi été accusés d'être à l'origine de problèmes sanitaires (en prétendant guérir des malades avec l'eau croupie des rizières par exemple), d'être des escrocs, mais surtout d'être parfois des instigateurs de désordres politiques. Depuis quelques années, au moins 
depuis 1992, la situation semble s'assouplir. «Semble » seulement car si quelques temples rouvrent, si de grandes cérémonies publiques sont organisées, tout se passe sous le contrôle de responsables reconnus par le gouvernement. De nombreux temples restent fermés ou interdits à la pratique, des cérémonies non autorisées. Des processions et prières aux entités sont autorisées. La possession reste officiellement proscrite mais les médiums transgressent facilement l'interdit sans être systématiquement sanctionnés. On ne peut donc pas parler d'une véritable libéralisation de cette pratique religieuse, tout au plus d'une certaine tolérance sous contrôle de l'État.

Cette partie, sur les relations sociales en œuvre dans le culte, éclaire la situation des fidèles et surtout des médiums. Ces derniers, en entrant au service des thánh, entament un processus que l'on peut qualifier de carrière au sens de l'anthropologie sociale ${ }^{20}$. L'expression " entrer dans la religion " qu'utilisent certaines bà dồng en France, illustre cet engagement crucial pris à un moment de leur vie. Orientant les activités sociales et atteignant la conception de soi, il compte parmi les éléments participant de l'identité des individus ; identité dans ses dimensions culturelle, religieuse, nationale même (au regard des héros historiques divinisés) à la mesure du milieu social et culturel environnant.

La migration amenant à changer de milieu, les éléments en jeu dans le culte restent-ils les mêmes?

\section{La situation de migration : le culte comme élément d'une nouvelle dimension identitaire}

En repérant comment s'exerce la pratique, quelles sont les permanences, les transformations, nous allons montrer qu'en changeant de contexte, le culte reste inscrit dans un certain rapport social, participe à la construction identitaire, mais dans une autre dimension que celle du pays. En nous appuyant sur la comparaison entre les conditions de pratique au Viêt Nam et en France, l'examen des conditions a minima du culte, c'est-à-dire de ses éléments fondamentaux, nous éclairera sur l'évolution du culte hors de son contexte de même que l'influence de la situation de migration. Les points d'ancrage autour desquels s'organise la pratique sont : un lieu, un temps, une musique, des acteurs ${ }^{21}$ . Si l'un de ces éléments vient à manquer le culte ne peut se pratiquer. Nous verrons cependant que la migration les affecte différemment.

\section{1 - Un lieu : le đền}

49 Au Viêt Nam, les cérémonies se déroulent dans des temples spécifiques, (đề $\left.{ }^{22}\right)$. Certains sont financés par des associations officielles de fidèles sous l'égide d'un ou d'une médium responsable (ông đồng đền ou bà đồng đền). Les constructions varient selon l'environnement et les moyens financiers. On aura ainsi soit des bâtiments à la façade richement ornée, encastrés dans le dense bâti urbain soit des petits temples de montagnes noyés dans la végétation et très fréquentés les jours de fêtes. On peut également trouver des temples privés, familiaux souvent, soit construits sur le terrain de la famille près de la maison d'habitation, voire mitoyens et communiquants, soit au dernier étage d'un immeuble. La famille, aidée par les fidèles du voisinage, en assure l'entretien. 
que soit le cas de figure, nous avons remarqué une nette séparation entre habitat et culte au Viêt Nam. Il en va autrement en France. D'une part, ce culte étant extrêmement marginal et les adeptes dispersés dans les différentes régions, les temples « ouverts » sont peu nombreux. L'initiative en revient principalement aux rapatriés d'Indochine qui, regroupés pour une part comme au C.A.F.I. de Ste Livrade dans le Lot-et-Garonne ou dans les corons du village de Noyant d'Allier, ont pu développer une vie sociale propre et conserver une pratique religieuse collective. Noyant d'Allier a accueilli une forte population de rapatriés d'Indochine et l'implantation vietnamienne y est encore très marquée. Une grande pagode construite en $1983^{23}$ en témoigne. C'est d'ailleurs dans l'enceinte de cette pagode, un peu à l'écart, qu'a été inauguré en octobre 1990, dans un pavillon à l'architecture française, le temple pour le culte des Thánh Mẫu. C'est aujourd'hui un haut lieu de culte et la plupart des bà đồng de France y sont allées ou en ont entendu parler. À Fréjus et Marseille également, les pagodes abritent dans leur enceinte, un espace réservé à ce culte. Notons que l'activité de chacun de ces lieux de culte dépend du dynamisme des responsables et selon les cas, on remarquera une plus ou moins grande effervescence.

\section{Une cérémonie au Viêt Nam}

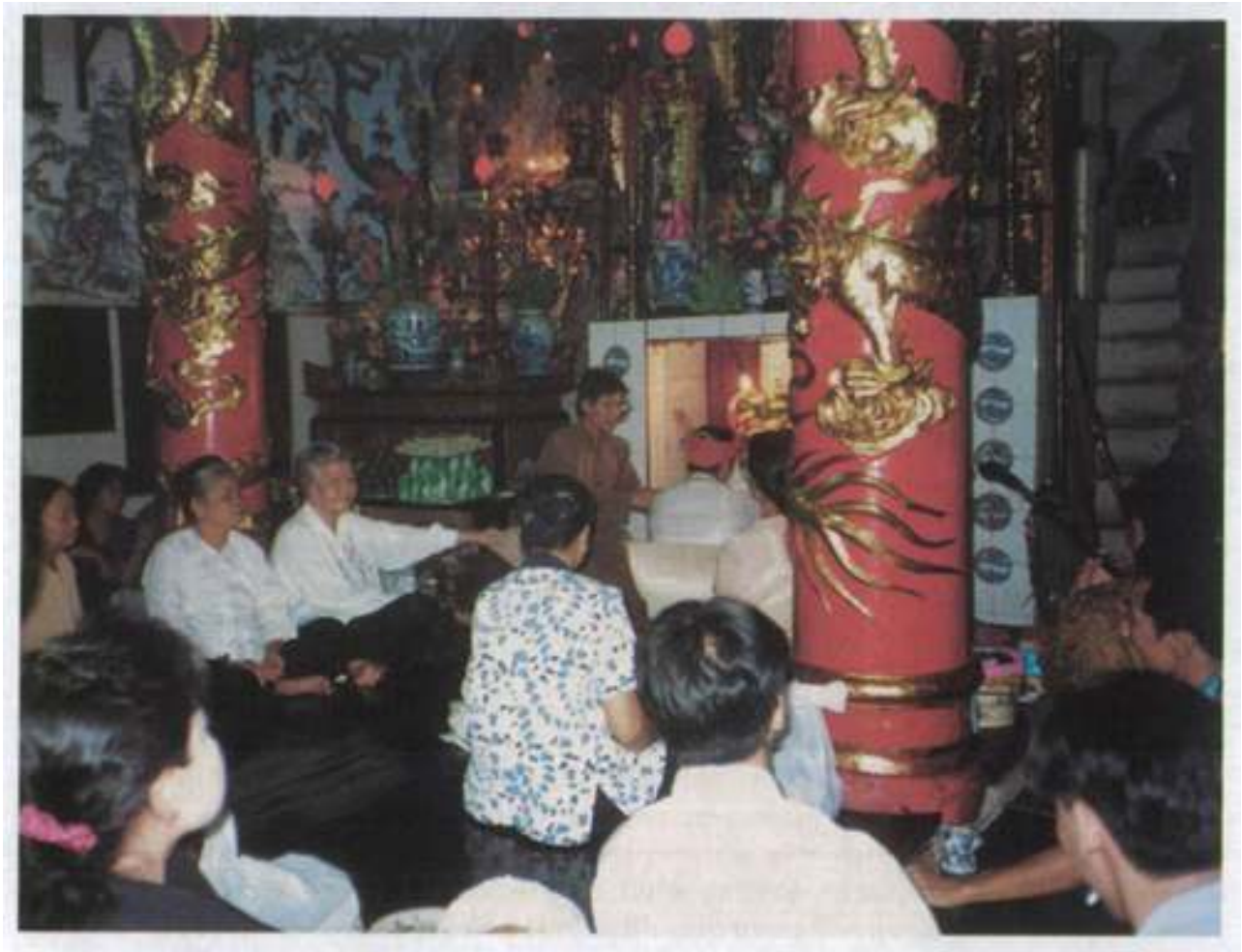

Hanoï, 1997

Dans la configuration que nous avons observée au Viêt Nam et en France en ce qui concerne les temples que nous avons qualifiés d'«ouverts", le temple comprend deux grandes parties : le lieu du culte lui-même et la cuisine. Les temples insérés dans l'espace d'habitat familial sont constitués du seul lieu de culte, la cuisine de la maison servant à la confection des plats clôturant la cérémonie. Dans la partie cultuelle, plusieurs autels sont édifiés, dédiés aux différentes catégories d'esprits, selon une organisation spatiale prédéfinie : un autel principal, central, est consacré aux "Saintes Mères », aux grands 
mandarins ; deux autels latéraux placés de part et d'autre et en alignement avec l'autel principal sont respectivement, à droite, l'autel du général Trần Hýng Đạoet à gauche, celui de l'imposante dame des montagnes accompagnée des demoiselles et des petits princes. Sous l'autel central, au niveau du sol, les serpents et tigres gardiens du temple ainsi que les «soldats» (lính) ont également le leur. L'organisation des autels dépend en partie de l'espace disponible, du nombre et de la taille des statues de thánh à placer sur les autels. Sur ce point, l'important n'est pas tant l'objet-statue que la représentation des thánh sur les autels, car elle atteste de leur présence. Les statues sont souvent des offrandes faites par les fidèles et elles sont consacrées lors d'une cérémonie au cours de laquelle on demande aux thánh de bien vouloir "descendre» dans les statues, d'accepter de les habiter. Il ne semble pas y avoir de règle stricte quant à leur taille ou leur nombre, et la richesse de la décoration, la taille des statues relèvent du budget disponible. Dans l'idéal, et surtout au Viêt Nam, les thánh ("Saintes Mères", mandarins, dames, princes demoiselles et enfants) ont leur statue installée sur l'autel correspondant. Représentations de faïence peinte mesurant entre trente et quatre-vingts centimètres environ, ces personnages, traits et parures, sont représentés tels que les légendes et les chants les décrivent : tenue traditionnelle de la Chine impériale pour les mandarins, les princes, et certaines dames; coiffes et tenues traditionnelles des régions des demoiselles. En France, dans le meilleur des cas, c'est-à-dire dans le cadre associatif, des statues ont été importées du Viêt Nam à grands frais, et leur nombre augmente au fil du temps et des dons, mais souvent, dans le cadre privé, faute d'espace ou d'argent, il faut opérer une sélection en respectant la hiérarchie. Remettant à plus tard l'éventualité d'honorer les autres thánh d'une belle statue, on s'ingénie néanmoins à leur offrir une représentation matérialisée sous la forme de poupées, jouets d'enfant revêtus des atours adéquats confectionnés de façon artisanale et les transformant ainsi en demoiselles ou petits princes. Dans les temples ouverts, un peu à l'écart, un autel est dédié aux donateurs décédés, élément que l'on ne trouve pas dans les temples familiaux. Notons que le culte des ancêtres n'est pas associé à l'espace du culte de possession quand celui-ci est pratiqué dans le logement familial en France. Un emplacement spécifique à demeure et distinct est réservé à l'autel des ancêtres, si possible dans une autre pièce. Il en va de même pour l'autel bouddhique. Le cadre de vie familial est ainsi traversé par les différents cultes. En cas d'espace restreint ou de moyens financiers limités, les autels sont adossés au mur du fond de la pièce, alignés, face à l'entrée principale. Dans le cas contraire, la répartition de l'autel central s'effectue sur deux plans : en premier plan, directement face à l'espace du rituel, l'autel des mandarins et en arrière plan, laissant un passage en intervalle, l'autel des saintes mères, plus sacré, donc à moindre portée des regards. L'espace intercalaire permet à la bà đồng de circuler entre les différents autels pendant le rituel et d'accéder à l'autel des saintes mères lors du nettoyage qui précède chaque cérémonie. Cette disposition sur deux niveaux est plutôt celle des temples "ouverts » viêtnamiens ou associatifs de France, alors que l'alignement simple est caractéristique des temples familiaux, en France. Un espace de quelques mètres carrés, couvert de nattes est réservé devant l'autel central (du premier plan si la configuration en comprend deux), pour le rituel effectué par la bà dồng. Les musiciens se placent à droite de ce même autel et le public se répartit sur le pourtour de la pièce, à même le sol, en veillant toutefois à ne jamais être dos à l'autel et à ne pas empiéter sur l'espace du rituel. Cette disposition se retrouve dans tous les cas observés. 
52 Les autels sont entourés d'un soin tout particulier. Chacun, supportant les représentations, (gravures ou statues) toujours hautes en couleurs, des thánh est richement orné de vases de fleurs, chandeliers, porte-encens, bols d'offrandes de fruits. $\mathrm{Au}$ plafond, au-dessus des autels, sont suspendus des objets votifs, accessoires des esprits, aux couleurs des différents palais : larges chapeaux viêtnamiens, et regroupés dans des mobiles de papier, fragiles confections sophistiquées aux teintes soutenues, les chaussures des demoiselles, des bateaux, des chevaux. Des tissus brodés et pailletés tendus devant les autels rehaussent encore l'éclat déjà rutilant de l'ensemble. Enfin, ralliant la modernité, des guirlandes électriques font parfois scintiller paillettes et cuivres de l'autel de leurs feux multicolores. L'impression qui se dégage de cet ensemble est celle d'un foisonnement : couleurs chatoyantes, brillances entourant les statues jusqu'à parfois les englober. La richesse et l'imposante présence des autels, sont telles qu'il est difficile au néophyte d'en distinguer les différentes composantes. Les murs de la pièce eux-mêmes, au Viêt Nam, peuvent donner lieu à un traitement particulier, quoique cela ne soit pas systématique. Ainsi, des fresques illustrant certains épisodes des légendes des thánh ou des divinités bouddhiques complètent la décoration du lieu. En France, dans les lieux de culte associatifs ou privés, de telles décorations n'existent pas. À l'extérieur du temple, à l'air libre, un espace est également investi pour le culte. On y trouve l'autel des âmes errantes et un four de briques servant à la crémation des placets, objets votifs, simulacres de papier offerts aux thánh, nécessaires à leur vie dans les hauteurs célestes.

Nous le voyons, l'espace du rituel a ses règles. En France, elles sont parfois difficiles à suivre, surtout dans le cadre familial. Au mieux, on réserve une pièce pour le culte, généralement la plus spacieuse, le salon-salle de séjour qu'on aménage de façon permanente en s'efforçant d'appliquer, autant que faire se peut, les normes d'organisation de l'espace religieux. L'espace commun ou de réception est alors transféré dans une autre pièce, une chambre, plus exiguë que la salle de séjour, sans que cela pose de problème particulier à la famille qui reproduit d'une certaine façon le mode d'habitat $\mathrm{du}$ pays d'origine où les pièces des habitations sont souvent moins spacieuses qu'en France. L'autel est installé au fond de la pièce, des étagères pour les offrandes aux thánh sont accrochées aux murs latéraux, un meuble sur le côté également, renferme la garderobe des thánh et un autre leurs accessoires (épées, hallebarde, rames, bâtons à grelots, drapeaux, etc.). Dans l'impossibilité de soustraire l'une des pièces du logement à l'usage ordinaire, on alterne deux pratiques dans le même lieu. La pièce, selon les circonstances sera salle de séjour ou lieu de culte. L'autel installé à demeure pour l'essentiel, est séparé de l'espace profane par un grand rideau que l'on ouvre le temps de la cérémonie, permettant ainsi aux habitants d'aller et venir en temps ordinaire, sans se soucier des règles de conduites à respecter lorsque l'autel est dévoilé24. Lors des cérémonies, c'est sur le buffet que sont disposées les corbeilles d'offrandes de fruits et légumes et autres objets, lọc qui seront distribués pendant la cérémonie. Et l'on sort d'un placard la petite table du rituel et ses accessoires (miroir, service à thé et verres) que l'on place devant l'autel central, après avoir déplacé table et chaises. Il y a donc, dans ce cas, une réorganisation de l'espace en fonction de l'utilisation, l'espace d'habitation restant sensiblement le même.

L'habitat français, en immeuble notamment, ne permet pas toujours de respecter les règles religieuses. Ceci vaut pour les adeptes du culte des thánh mais aussi pour les bouddhistes orthodoxes. En effet, suivant la norme, les autels doivent être placés sur la partie la plus élevée de l'habitation, la plus proche du ciel et donc au plus loin du monde 
profane. Or, dans les appartements en immeubles, cette règle ne peut être respectée que si l'on occupe un logement au dernier étage. Dans le cas contraire, alors que l'on aura pourtant pris soin de le placer le plus haut possible dans le logement, les voisins du dessus « marcheront » eux, impunément, sur l'autel. Il n'est guère d'autre solution à ce problème que d'ignorer l'étage du dessus. L'usage de l'encens ne va pas non plus de soi car la fumée odorante s'insinue très facilement dans les constructions modernes, comme j'ai pu le constater, à mon arrivée sur le palier de l'étage, le jour d'une cérémonie. Certaines personnes ont donc réduit la consommation d'encens pour ne pas incommoder les voisins. La question du four est plus délicate à résoudre. Lorsque les appartements sont agrémentés d'un balcon, on y installe l'autel des âmes errantes, mais pas le four, trop peu discret. En fait, celui-ci, le plus souvent un grand bidon de récupération muni d'un couvercle, est placé directement dans l'appartement, la cuisine ou le couloir, et l'on veille à ce que la crémation des placets et objets votifs s'opère sans trop de fumée, ce qui n'est guère difficile puisque un brasier de papier bien entretenu se consume en flammes claires. Enfin, les conditions d'habitat collectif entravent l'usage de cet élément indispensable à toute cérémonie qu'est la musique. Si au Viêt Nam, ou en France dans les lieux de culte isolés, la musique bat son plein, occupant largement l'espace sonore, dans les immeubles, les voisins ne trouvent pas toujours à leur goût les hymnes et les airs que diffuse le magnétophone pendant les cinq à sept heures que dure une cérémonie. Bien que les adeptes, médiums et assemblée essaient d'être des plus discrets, le bruit traverse les cloisons minces, et il arrive que l'occupant du dessus ou du dessous manifeste par quelques coups sur la paroi son mécontentement. Le volume sonore est alors immédiatement diminué, les conversations baissent d'un ton et la bà đồng évolue en atténuant les frappements au sol des talons, comportement caractéristique de quelques thánh. 


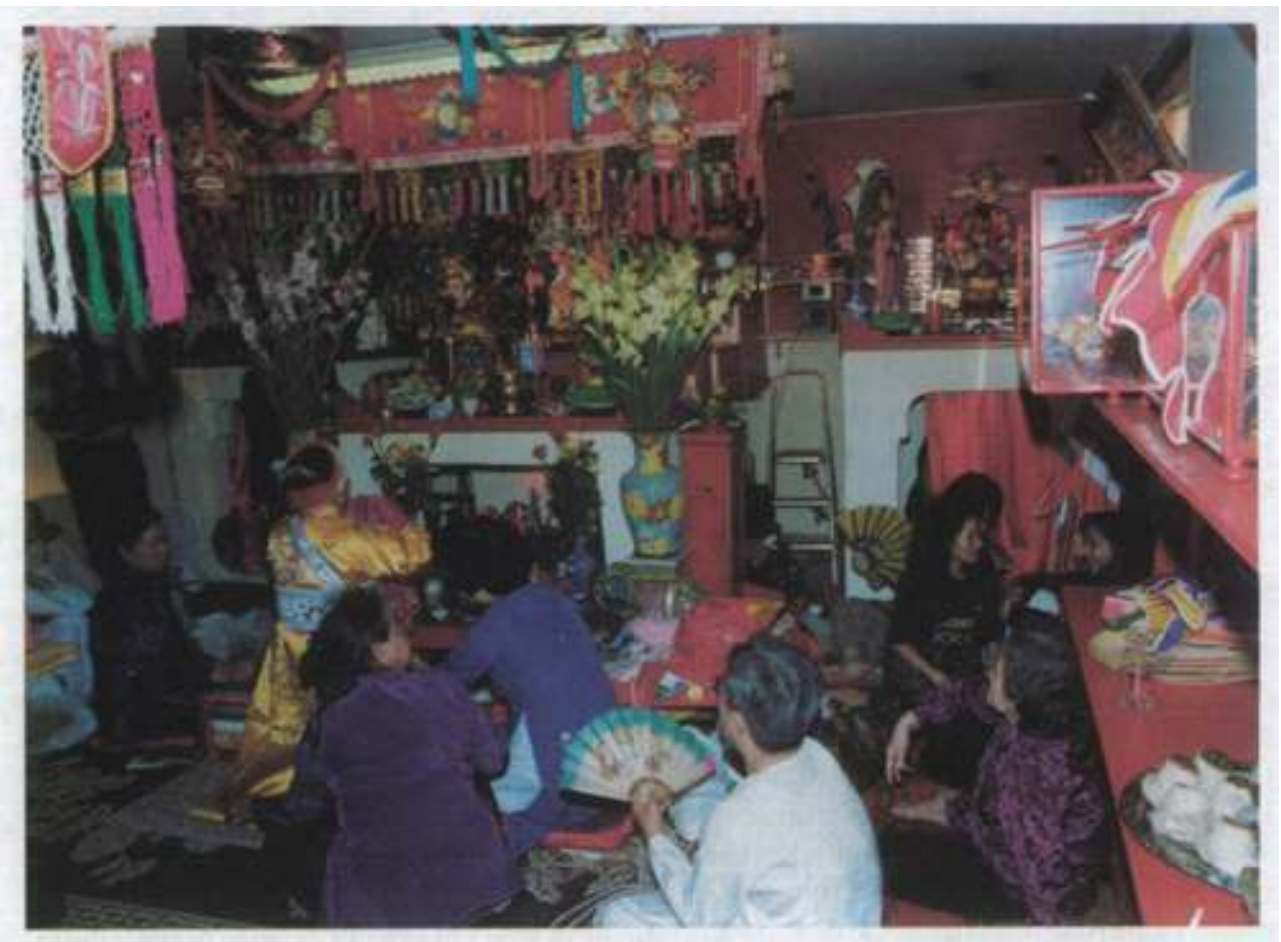

- Le Thánh, face à l'autel accomplit le rituel ;

- À ses côtés, les assistantes ;

- En retrait, les invités :

Noyant d'Allier, 1995.

Nous voyons, ainsi, que la situation de transplantation, sans empêcher la mise en place de lieux de culte impose un certain nombre de modifications, restrictives pour la plupart. L'espace et les ressources amènent une relative diversité dans les aménagements des autels et, en fait, les bà đồng qui n'ont pas réussi à installer à leur domicile un autel aux thánh et qui sont obligées d'aller exercer le culte chez d'autres sont peu nombreuses. La première condition de pratique, le lieu, étant remplie de façon relativement satisfaisante, qu'en est-il de celle du temps?

\section{2 - Le temps du culte}

Le calendrier du culte, fondé sur le calendrier lunaire, rythme la vie religieuse tout au long de l'année. L'année rituelle comporte des cérémonies d'ouverture et de fermeture et certaines périodes charnières comme « l'entrée de l'été » ou « la sortie de l'été », le début de l'année lunaire, sont des moments privilégiés pour les cérémonies ainsi que les dates de commémoration spécifiques à certains esprits. D'autres périodes comme le septième mois lunaire, consacré aux morts, ou le mois précédent le nouvel an lunaire, sont sans cérémonies. Certains jours sont considérés comme fastes, d'autres néfastes et l'on essaie, autant que possible, de respecter ce calendrier. Sans entrer dans les détails du calendrier religieux, il suffira ici d'en connaître l'existence et de voir comment il en est tenu compte en France. Si les périodes de suspension des cérémonies sont scrupuleusement respectées, et on comprend aisément que le respect des interdits de pratique ne pose pas de problème particulier, en revanche, il est parfois difficile d'honorer le calendrier lorsqu'il 
s'agit de la pratique à date fixe. En effet, si les jours propices sont en pleine semaine, une partie de la population concernée peut difficilement se dégager des occupations, professionnelles ou familiales, pour participer aux cérémonies. La bà đồng elle-même peut se trouver dans ce cas. Au Viêt Nam l'organisation sociale semble plus souple, les personnes officiant lors d'une cérémonie avaient pris un congé pour cette occasion. En France, le problème est résolu d'une autre façon. La bà đồng demande aux thánh de bien vouloir accepter le report de la cérémonie au samedi ou dimanche suivant, jour de repos de la bà dồng si elle travaille, et qui permettra la présence d'une plus nombreuse assistance. À notre connaissance, la dérogation ne pose pas de problème. Ainsi, nous voyons là aussi que la vie dans le contexte de transplantation gêne les conditions normales de la pratique religieuse, risquant de la compromettre. Cependant, l'instauration d'une certaine souplesse, permet de franchir les obstacles du calendrier tout en conservant un temps légitime à la pratique.

\section{3 - La part de la musique}

57 Nous avons évoqué le lieu et le temps comme conditions nécessaires à la pratique du culte. Nous avons vu par quels aménagements les adeptes réussissent à surmonter les obstacles sur ces points, dus à la situation de transplantation. Ces deux premiers aspects, bien qu'importants pour la pratique, ne posent pas de réelles difficultés et les modifications ne semblent pas déranger les acteurs qui disent s'en accommoder, même si, disent-ils, les conditions ne sont pas idéales.

La question de la musique est autrement plus importante. Si l'on compare les conditions du culte à une bâtisse en construction, le temps et le lieu font figure de murs simples alors que la musique en serait un «mur porteur ", un pilier sur lequel repose une bonne partie de l'édifice. L'analyse de Gilbert Rouget sur la place de la musique dans les cultes de possession se trouve ici tout à fait confirmée ${ }^{25}$. Elle est un élément constitutif de toute cérémonie et nous verrons que paradoxalement, bien qu'élément de base, c'est pourtant ce qui donne lieu à la transformation la plus remarquable, à la fois par rapport à la pratique actuelle au Viêt Nam et celle observée en France dans les années soixante.

Toute cérémonie se déroule sur un fond musical quasiment ininterrompu du début à la fin. L'accompagnement musical se fait sous des formes instrumentales ou chantées, et dans certains cas (pour le dixième prince, par exemple, particulièrement sensible à la musique), une personne de l'assistance chante a capella. Un air instrumental ou chanté particulier correspond à chaque étape du rituel : air d'invocation lorsque la médium, la tête et les épaules couvertes d'un voile rouge, attend la « descente » d'un esprit, hymne ${ }^{26}$ spécifique pour chacune des catégories de thánh, airs des prosternations, des "danses » des thánh. Bien que les hymnes, soient la plupart du temps dédiés à des personnalités précises du panthéon, le même chant peut être attribué à deux thánh différents, en fait deux aspects d'une même entité, et même, certains textes, plutôt que personnels, s'adressent à un ensemble de thánh en correspondance: hiérarchique (mandarin ou prince par exemple) ou numéro d'ordre (deuxième dame, deuxième demoiselle). Le corpus des hymnes ne semble pas établi de façon définitive. Les premiers textes semblent avoir été écrits au XIX siècle mais de nombreux autres, variations sur un même thème, l'ont été récemment ${ }^{27}$. Les hymnes sont ainsi l'objet d'une création continue, des chanteurs composent de nouveaux textes poétiques et enrichissent ainsi le corpus au fil du temps. Le culte n'étant pas bâti sur un dogme rigide, les chanteurs des différents 
orchestres (cung văn) dès lors qu'ils respectent la métrique spécifique aux hymnes peuvent laisser libre cours à leur inspiration.

Les musiciens jouent un rôle extrêmement important dans le déroulement de la cérémonie. Traditionnellement, l'orchestre est constitué de trois personnes : un joueur de tambour, un violoniste (violon traditionnel chinois, à deux cordes), un guitariste (instrument traditionnel ou guitare moderne). Mais les musiciens peuvent être plus nombreux, nous avons ainsi l'exemple d'un groupe de six personnes et l'on aura une flûte, des cliquettes, ajoutés à l'ensemble de base. L'orchestre peut être mixte et les chants sont souvent interprétés par l'un des instrumentistes. Au Viêt Nam, ils sont professionnels et sont engagés à chaque cérémonie. Ils viennent avec leurs instruments un peu avant la cérémonie et repartent après le repas final. Ils suivent musicalement, étape par étape le rituel, s'ajustant à chacun des faits et gestes du génie incarné, suivant son bon plaisir. Or, le temps de chaque incarnation varie, de même que les différents moments qui la composent : les princes aimant à s'attarder à écouter la musique, certaines demoiselles dansant parfois de longs moments. Les musiciens, réagissant au moindre changement d'attitude de la bà đồng, sont complètement « pris » par l'action, contrairement au public ordinaire qui peut aller et venir, sans trop se préoccuper de l'action en cours. Si les musiciens amateurs peuvent être des adeptes ou des médiums, les professionnels le sont rarement. Notons à ce propos que les possessions spontanées, fréquentes parmi les personnes de l'assemblée et les assistants, n'arrivent jamais aux musiciens pendant leur prestation. Entre la bà đồng et l'orchestre s'établit une relation privilégiée, reposant sur de la complicité. Les musiciens sont parmi les premiers à être gratifiés par les thánh, et généreusement. Parfois, un thánh charmé par un hymne à sa gloire qu'il juge particulièrement bien interprété, lance avec enthousiasme du côté de l'orchestre une volée de pièces ou des billets supplémentaires. Les regards, hochements de tête, sourires échangés de part et d'autre au cours de la cérémonie, rendent également compte de cette relation de proximité.

61 En France, la situation diffère considérablement car il n'existe pas actuellement de personnes formées pour ce type de prestation. Les cérémonies décrites, dans les années soixante, dans le Lot et Garonne étaient accompagnées par un orchestre amateur il est vrai, mais bien présent, constitué de bà đồng qui palliaient ainsi le manque de spécialistes. Or, aujourd'hui, la plupart de ces femmes sont décédées ou sont trop âgées pour tenir ce rôle et aucune relève ne s'est manifestée. Cet obstacle particulièrement important a été en partie contourné par le recours à la technologie; la musique et les chants sont des enregistrements que l'on diffuse à l'aide d'un magnétophone. Ainsi, l'orchestre de base, en France, est-il constitué d'une cassettothèque, d'un magnétophone, et d'un(e) technicien(ne). Ainsi, pour présenter une prestation musicale enregistrée satisfaisante, il faut avoir surmonter au moins cinq grandes difficultés.

\section{a - La constitution d'une cassettothèque}

Même si l'on tient compte des correspondances possibles entre certains hymnes, et même si tous les thánh ne s'incarnent pas, la collection doit contenir les airs de chacun, au cas où, ainsi que les différentes musiques d'invocations, de prosternations. Les cassettothèques des bà đồng en France, en comprennent de soixante à trois cents pour les plus complètes. L'initiative des premiers enregistrements revient aux médiums ellesmêmes, (celles de Ste Livrade), qui avaient anticipé la carence en musiciens en s'enregistrant. Aujourd'hui, on fait parvenir du Viêt Nam les enregistrements ou l'on 
copie soit des cassettes prêtées, soit le son des vidéos que certaines bà tồng ont réussi à sortir du pays lors de séjours au Viêt Nam.

\section{b - La qualité des enregistrements}

Plusieurs facteurs entrent en jeu dans la qualité. Le travail technique d'abord, avec la prise de son parfois mal faite ; les conditions de la prise de son ensuite, avec le parasitage de l'ambiance de la cérémonie au cours de laquelle a été fait l'enregistrement. Enfin, la multiplication des copies de cassettes audio ou le « repiquage" du son vidéo altèrent aussi souvent la qualité du son et, si en fin de compte, on reconnaît toujours les airs, la comparaison avec un orchestre véritable n'est pas soutenable. Des musiciens professionnels, au Viêt Nam, ont remédié en partie à ces problèmes de qualité en proposant aux bà đồng de France, des cassettes enregistrées sur commande, hors de tout contexte de cérémonie. Toutefois, cette pratique est très onéreuse et les adeptes de France y ont aujourd'hui d'autant moins recours que l'on peut se procurer, à très bon marché et en vente libre, des cassettes enregistrées des văn.

\section{c - La durée des enregistrements}

La durée est un point saillant qui influe sur le bon déroulement du rituel. Les cassettes ne sont pas enregistrées in extenso. Chaque face comprend un seul air ou chant et selon une durée qui varie de quelques minutes à plus d'une dizaine de minutes, selon les cas. Les enregistrements étant souvent effectués sur le vif lors d'une cérémonie, les durées sont celles des séquences de possessions de cette cérémonie. Or, d'une médium à l'autre ou d'une cérémonie à l'autre, la durée de l'incarnation varie. Si, à l'occasion, la durée de l'enregistrement d'un air suffit à accompagner la prestation dansée d'un thán ${ }^{28}$, ce ne sera pas le cas à la cérémonie suivante. Pour que le thánh puisse aller jusqu'au bout du temps de danse, qu'il a voulu plus long cette fois, il faudra rembobiner une partie de la bande. Une manipulation relativement fréquente et qui, aussi courte soit-elle, perturbe l'ambiance. Dans l'intervalle de rembobinage, lorsque le temps de coupure est très court, la bà đồng incarnant le thánh, continue sa prestation, mais dans le cas contraire, elle patiente debout sans bouger, toujours possédée par le génie qui montre des signes d'impatience si la manipulation se prolonge; les évolutions ne reprennent alors qu'avec le retour de la musique.

\section{d- Un appareil adapté}

Les appareils utilisés par les bà đồng en France vont du simple au sophistiqué. Il faut au minimum un appareil muni de deux lecteurs de cassettes, car pour éviter les coupures, on doit pouvoir préparer sur l'un des lecteurs la musique suivante pendant que l'autre diffuse celle appropriée au moment du rituel. Il semble difficile de mener à bien la partie musicale sans double lecteurs, les morceaux ne s'enchaînant pas, les temps de coupure se multiplient. Quelques médiums ont fait l'achat d'un système de sonorisation plus sophistiqué. Une table de mixage facilite les enchaînements, un casque permet de chercher et de caler les morceaux choisis, une fonction de préécoute discrète limite les risques d'erreur de cassette ou de démarrage de la diffusion sur l'amorce de la bande et enfin, placés de part et d'autre devant l'autel, des haut-parleurs inondent la pièce de musique. 


\section{e - Un(e) technicien(ne) chevronné(e)}

66 la cérémonie. La population des bà đồng étant essentiellement féminine en France, ce sont surtout des femmes que l'on trouve aux commandes des appareils. L'attention soutenue est peut-être le seul point commun avec le groupe de musiciens traditionnels, encore arrive-t-il que la personne quitte son poste quelques minutes de temps à autre quand la bande défile ou qu'une autre personne la relaie. Comme les musiciens, la personne préposée aux appareils doit suivre les différentes étapes du rituel et ne peut se laisser distraire. À elle d'installer, de vérifier le matériel avant la cérémonie, de préparer les cassettes, de faire en sorte que les musiques s'enchainent de façon harmonieuse, et veiller au niveau sonore (les enregistrements provenant de sources différentes, les niveaux sonores ne sont pas harmonisés). La comparaison avec l'orchestre présent trouve rapidement ses limites car à la différence de l'orchestre, la technicienne est souvent en décalage avec l'action puisque, lorsqu'elle prépare les cassettes, elle anticipe une partie du rituel. Chaque séquence de possession est accompagnée de trois rythmes musicaux différents : la prise de possession, les prosternations et l'air spécifique du thánh incarné. Trois manipulations de cassettes par incarnation sont donc nécessaires. Bien que l'ordre des incarnations soit préétabli par la hiérarchie, l'occurrence relative des incarnations de certains thánh, et l'inégale longueur des séquences de possession rendent impossible la programmation à l'avance de l'accompagnement musical d'une cérémonie. Les cassettes sont l'objet d'un soin particulier : répertoriées, classées et rangées sur des étagères ou dans des mallettes spéciales, aménagements qui les rendent immédiatement disponibles.

La technique est une lourde tâche accomplie le plus souvent par une personne qui n'avait pas forcément de compétences en la matière, tel le mari déjà âgé de cette bà đồng, agriculteur plus au fait, a priori, du maniement de la charrue que de celui des appareils «Hi Fi ». Selon les personnes, le matériel, les moments, la prestation est plus ou moins réussie. Si les maladresses soulignent le manque d'habitude de certaines personnes, d'autres sont devenues très compétentes, aussi bien dans la rapidité et la précision des gestes. Paradoxalement, le(la) technicien(ne) est la personne la plus attentive au déroulement de la cérémonie mais une bonne partie de cette attention est orientée, en fait, vers les aspects techniques. La relation de complicité précédemment soulignée entre la bà đồng possédée et l'orchestre est ici absente. Pourtant, et c'est une attitude traditionnelle qui perdure, pendant toute la cérémonie la personne diffusant les cassettes est assimilée aux musiciens et les thánh la gratifient de la même manière, récompensant un morceau bien exécuté ou un hymne chanté avec brio.

La conclusion de ce point sur la musique est, si je puis dire, marquée d'un bémol car malgré la virtuosité, la compétence des techniciens ou la qualité des enregistrements, les médiums reconnaissent ne pas être pleinement satisfaits de cet aménagement. Les musiciens sont regrettés de façon unanime et les médiums estiment de bien meilleure qualité, tant sur le plan pratique que spirituel, les cérémonies accompagnées par un véritable orchestre.

\section{5 - Les acteurs}

69 Les acteurs sont évidemment les humains : médiums et fidèles, mais j'inclurai également les thánh dans cette catégorie " acteurs ». C'est là un parti pris méthodologique car dans 
la réalité, la parole des esprits passe par les médiums. Mais les thánh étant perçus comme acteurs à part entière de la situation par la population, la distinction permet de mieux situer la place qu'ils occupent dans le nouveau contexte.

\section{a - Les humains}

Situons d'abord médiums et fidèles d'un point de vue démographique. Au Viêt Nam, le culte est populaire mais ne concerne qu'une partie de la population. Elle est relativement nombreuse, plusieurs milliers de personnes d'une même région. Malgré les restrictions de la législation, cette concentration démographique demeure un atout pour les regroupements. Les personnes se retrouvent en réseaux de connaissance, souvent sous l'égide des ông đồng đền ou bà đồng đền (médiums responsables de temples). De plus, la proximité géographique et une gestion du temps relativement souple au Viêt Nam permettent aux personnes de participer à différentes cérémonies. En France, la pratique est le fait exclusif d'une population d'origine viêtnamienne, sinon de nationalité, en tous cas de culture. Celle-ci constitue une composante démographique minoritaire, d'où le nombre, proportionnellement très restreint de médiums; tout au plus pouvons-nous estimer à moins d'une centaine l'ensemble des médiums au service des thánh et évaluer de trois à quatre cents personnes l'ensemble des fidèles. Ceci dit, ces chiffres ne sont qu'une estimation. Mais il est essentiel de garder à l'esprit le nombre restreint de personnes concernées par cette pratique. Aux inconvénients du petit nombre, s'ajoute l'effet de la dispersion. La population habite dans différentes régions, mais c'est en région parisienne et dans les grandes villes que l'on compte le plus grand nombre de membres. Il y est plus facile d'entretenir des réseaux de connaissance. Les membres résidant dans les petites villes se disent isolés et n'ont d'autre possibilité que de se déplacer pour se joindre à une collectivité un peu plus large. Les déplacements s'effectuent de façon individuelle pour les cérémonies ordinaires ou organisées dans des cadres privés. Pour les grandes fêtes annuelles, un bus est quelquefois affrété qui emmène les participants pour une sortie de deux ou trois jours. Ceci ne vaut que pour le cas du temple de Noyant d'Allier, conçu pour héberger une partie des membres (les autres étant accueillis par des rapatriés d'Indochine habitant dans les maisons à proximité). Les cérémonies sont, dans ce cas, des occasions de rencontres, retrouvailles entre personnes de même culture, éloignées géographiquement. Pendant et après la cérémonie, dans la pièce voisine, les uns sortent les cartes à jouer typiques des jeux viêtnamiens, pour des parties qui dureront tard dans la soirée, pendant que les conversations des autres vont bon train autour d'une tasse de thé vert. C'est un moment de vie collective particulièrement apprécié des participants. D'autre part, la place du culte dans la société globale accentue l'isolement de la population, diminuant les chances d'un recrutement plus large et peut-être celles du maintien de ce culte.

71 À la différence du bouddhisme orthodoxe, le culte des Thánh Mẫu est exclusivement ancré dans la collectivité culturelle d'origine. En effet, s'il n'est pas rare de rencontrer des Occidentaux adhérant au bouddhisme dans les pagodes en France, ils sont plus rares dans les cérémonies des Thánh Mẫu, plus en retrait. Et leur présence n'atteste pas une réelle participation au culte. Dans une relation de proximité avec le culte, le cas de figure le plus courant est celui de l'union mixte. C'est généralement soit le conjoint français d'une bà đồng soit celui d'une personne de la famille de la bà đồng. La plupart du temps, leur connaissance du pays se limite à ce qui leur est apporté par la bà đồng et sa famille. Ce sont aussi parfois d'anciens militaires d'Indochine mariés à la compagne rencontrée là- 
bas et rapatriée en 1954. Dans une relation plus distanciée, on rencontre les Européens invités à l'occasion de grandes cérémonies annuelles associant des processions dans les rues. Ces manifestations sont rares en France et ne se rencontrent que dans une seule commune, à ma connaissance. À cette occasion, les élus locaux et amis de la commune sont conviés au repas collectif où une table leur est réservée. Certains, restent encore un moment après le repas et, affichant un air d'incompréhension, contemplent le rituel de la pièce voisine, ouverte sur l'espace cérémoniel. Plus encore que pour les conjoints, la présence ponctuelle de ces spectateurs est l'expression de l'estime et de remerciements envers les organisateurs, plutôt qu'un intérêt religieux. Dans les deux cas, ils constituent une très faible fraction du public. Parmi les invités, ce sont des spectateurs, et non des participants. Pour ces personnes, le culte est perçu comme une pratique traditionnelle viêtnamienne, en lien avec le bouddhisme, propre à la culture des personnes de cette origine dont ils respectent la religion sans y adhérer.

Le culte ne perdure que s'il s'assure un recrutement. Le contexte culturel français joue un rôle non négligeable dans la transmission, le renouvellement des effectifs. Nous avons évoqué une relève "timide " du côté des "deuxièmes générations ». Il est hasardeux là encore de donner un chiffre. Les médiums initiés, ou seulement appelés, nés ou arrivés enfants en France sont en petit nombre. Dans l'état actuel de mes connaissances, quarante personnes environ ont accompli la cérémonie d'initiation, Đội bát nhang(lit. "porter sur la tête les bols de baguettes d'encens »): trente en région parisienne, dénombrées sur la base des informations fournies par le ông đồng đền qui a présidé à ces initiations, et des bols à encens des cérémonies d'initiation de chacun, conservés au temple et rangés sur des étagères. J'ai, d'autre part, recensé personnellement une dizaine de personnes initiées, dont neuf de la même famille, fait à souligner. Cette question du renouvellement des médiums, acteurs principaux, est à relier avec la place de la langue dans le culte. La parole y joue un grand rôle, les esprits s'exprimant dans la langue, selon les accents de leur terroir. Même si le langage est en partie construit à partir de formules stéréotypées, la connaissance du viêtnamien est indispensable pour le bon déroulement du rituel. Or, l'usage de la langue évolue selon les générations. Les personnes de la deuxième génération en ont encore un usage courant, mais avec une accentuation parfois déficiente et un vocabulaire appauvri. Pour la génération suivante, la connaissance linguistique ne dépasse pas, la plupart du temps, une compréhension superficielle et l'emploi de quelques expressions rudimentaires. Comme d'autres aspects du culte qui ont $\mathrm{du}$ se transformer pour perdurer, il y a fort à parier que la langue du rituel va aussi être l'objet de changements permettant ainsi l'intégration de nouveaux acteurs. Déjà, il est remarquable que la verve des thánh soit en rapport proportionnel direct avec la connaissance de la langue du médium. Les nouvelles générations de médiums ont également une approche nouvelle du culte. À la différence des plus âgés qui en avaient surtout une connaissance pratique et peu théorisée, certains des nouveaux bà đồng et ông đồng, s'intéressent aux écrits sur le culte. Ils trouvent, notamment dans les travaux des ethnologues, des éléments de compréhension voire de reconstruction du panthéon. C'est là un usage paradoxal de leur travail auquel n'avaient certainement pas pensé les auteurs. Les écrits sont critiqués sur certains points mais ils sont une base de connaissance et prennent parfois une valeur de preuve dans la légitimation du culte. Comme dit cet ông đồng à propos de deux ouvrages publiés au Viêt Nam (une étude ethnographique et un recensement des chầu văn) : «ces livres, c'est très important, c'est très précieux, comme ça les jeunes voient que c'est une vraie religion! C'est notre bible!». Il est difficile d'analyser l'adoption ou le rejet de la pratique. Les quelques exemples recueillis montrent 
des situations diverses tant sur le plan du capital culturel des personnes, que de leur situation socio-économique, mais les données sont pour l'instant trop fragmentaires et se révèlent insufisantes pour une affirmation étayée.

En se référant aux travaux passés réalisés dans le champ des relations interethniques, sur les phénomènes d'acculturation, on peut cependant émettre une hypothèse. Celle-ci est fondée sur la forme d'acculturation des différentes générations. À la différence des personnes des premières générations, dont l'acculturation est matérielle, c'est-à-dire de surface, préservant les normes et valeurs de leur culture d'origine, les jeunes sont dans un processus d'acculturation formelle ${ }^{29}$ qui atteint l'individu dans sa dimension la plus profonde, sa structure mentale. Ainsi, le mode de vie en France, et surtout l'acculturation des jeunes générations à la société française sont des freins à la continuation du culte, tout au moins sous sa forme actuelle

\section{b - Les entités spirituelles}

74 Les thánh eux-mêmes, enfin, subissent les effets de la migration de leurs adeptes. Selon les médiums, "ils sont les mêmes mais ils sont différents en France ». Le panthéon est le même que celui des cérémonies du nord du Viêt Nam, les thánh sont présents ${ }^{30}$, descendent sur les médiums, protègent, conseillent dans les cérémonies, se manifestent toujours dans les rêves des médiums, mais n'ont plus tout à fait la même nature qu'au pays. Ici, ils sont plus tolérants, plus conciliants, ils acceptent des conditions d'incarnations moins strictes. Nous en avons pour preuve la manière dont les thánh limitent les manifestations bruyantes pour ne pas gêner les voisins. Nous avons également évoqué précédemment les aménagements du lieu, du calendrier et de la musique. Ils descendent sur leurs servantes, les «habitent» mais ce n'est pas avec la même « force ", ni avec les mêmes exigences que sur le territoire viêtnamien disent les bà đồng les plus âgées qui ont connu la pratique au Viêt Nam. C'est aussi le sentiment d'une jeune bà đồng, élevée et initiée en France qui est allée au Viêt Nam et qui a pu y organiser une cérémonie. «Là-bas, dit-elle, c'est plus fort, quand je suis allée dans la partie la plus sainte du temple (où se trouve la statue d'une Thánh Mẫu), j'avais les larmes qui coulaient sans m'en rendre compte, je ne pouvais pas m'en empêcher ! ». La Sainte Mère, selon elle, manifestait ainsi la satisfaction de la visite de sa servante : «c'était comme une mère qui retrouve sa fille... » commente la jeune femme encore émue deux ans après. De plus, ajoutent les médiums, il n'y a pas de manifestations spontanées en France, alors qu'au Viêt Nam, il leur arrivait d'intervenir directement, hors du cadre cérémoniel, lors d'événements graves. Par exemple, l'un des grands mandarins du panthéon (le cinquième, entité particulièrement importante) descendu, de façon inopinée, sur une fillette pour prévenir et apporter son aide dans l'accouchement prématuré de sa mère.

En conclusion, les transformations de la pratique et le discours qui l'accompagne éclairent sur la place des médiums en France et sur le changement de sens dont ils font l'objet en changeant de contexte global. Au Viêt Nam, les médiums occupaient une position sociale plutôt valorisante, à deux niveaux. Par leur milieu social d'une part, car le culte nécessitant des dépenses non négligeables, il était surtout pratiqué par des personnes disposant de revenus confortables, souvent des femmes de la bourgeoisie. D'autre part leur rapport privilégié aux entités assurait aux médiums un certain ascendant sur les fidèles. Par l'intermédiaire des thánh, ils se présentaient comme détenteurs d'un pouvoir magico-thérapeuthique (de protection, guérison et divination). Pouvoir qui fut à plusieurs reprises interprété en terme d'influence néfaste, notamment 
politique, pour la population par les gouvernants. Les interdictions gouvernementales qui frappèrent ou restreignent encore la pratique du culte, montrent les intrications entre le religieux et la vie sociale, dans cette dimension essentielle qu'est le politique.

En France, le culte des Thánh Mẫu s'inscrit dans un tout autre rapport à la société globale.

L'élément qui structure ce rapport est la situation minoritaire des membres du culte. Une minorité démographique, bien sûr, qui ne favorise pas le développement d'une pratique requérant un minimum de collectivité, mais surtout une situation sociologique minoritaire. Le pays est différent à tous points de vue, tout particulièrement sur le plan religieux. Excepté pour les catholiques viêtnamiens qui trouvent en France une familiarité religieuse, les autres expressions religieuses du Viêt Nam sont absentes du champ religieux français ou représentées de façon marginale. Le bouddhisme, grande religion d'Asie, est mieux représenté mais reste minoritaire. Pour ce culte, inexistant en France avant l'arrivée des rapatriés et encore très peu connu hors du cercle des familiers du culte, tout était à construire. Les fidèles ont repris la pratique, d'abord, de façon discrète, dans les logements, puis ils ont pu disposer d'un espace pour le rituel dans l'enceinte des pagodes à Fréjus et Marseille, enfin, en 1988 ils ont fait construire, à Noyant d'Allier, un bâtiment réservé au culte. Ils se revendiquent avant tout bouddhistes ce qui était déjà le cas dans le passé, mais cette affirmation est renforcée, aujourd'hui, par la crainte d'être assimilés à une secte. En même temps, ils se rapportent à une tradition et à une culture viêtnamienne, "c'est notre tradition, notre culture à nous » disent-ils. Ces déclarations sont surtout faites à l'intention des étrangers au culte, a fortiori les Occidentaux, parfois invités, et qui ont du mal à comprendre ce qui se passe. Mais elles sont aussi intégrées à une redéfinition identitaire en terme d'ethnicité, à l'intention des jeunes générations.

Ainsi, pour perdurer dans le cadre français, le culte évolue. Dans la pratique d'une part en s'adaptant matériellement aux nouvelles conditions de vie, et dans les fondements d'autre part, la question du pouvoir, centrale au Viêt Nam, s'efface pour laisser la place à une problématique plus culturelle et plus précisément, orientée vers l'ethnicité. Autour de la cérémonie s'opèrent des regroupements qui favorisent l'extériorisation des traits culturels viêtnamiens (parler, manger, prier, activités de loisirs) et participent ainsi d'une certaine communalisation. À l'heure actuelle ce n'est pas tant la puissance que l'on requiert des thánh que leur présence. Ainsi, dans la migration, les thánh, ont comme mission implicite le maintien d'une identité ethnique.

\section{BIBLIOGRAPHIE}

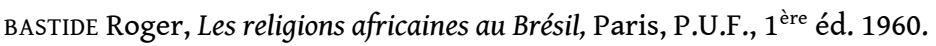

CADIÈRE Léopold, Croyances et pratiques religieuses des Vietnamiens, 3 tomes, Paris, E.F.E.O, réimp. 1992.

DE HEUSCH Luc, Pourquoi l'épouser ? et autres essais, Paris, Gallimard, 1971.

DURAND Maurice, Techniques et panthéon de médiums viêtnamiens, Paris, E.F.E.O, 1959. 
GOFFMAN Erwin, Asiles. Étude sur la condition sociale des malades mentaux, Paris, Éditions De Minuit, 1968.

ROUGET Gilbert, La musique et la transe. Esquisse d'une théorie générale des relations de la musique et de la possession. Paris, Gallimard, nouvelle édition revue et augmentée, 1990 (coll. « Tel »).

SIMON Pierre Jean, SIMON-BAROUH Ida, « Hâù bóng, un culte viêtnamien de possession transplanté en France », Cahiers de l'Homme, 1973.

THAN Huyên, «Aperçu sur les croyances et religions au Vietnam » in Études Vietnamiennes, $n^{\circ} 51$ (nouvelle série) spécial « Les religions et croyances populaires au Vietnam », Hanoi, 1996, pp. 5-22.

NGo Duc Thinh, «Le culte des Génies féminins et des Mères-Déesses "Mâu" » in Études Vietnamiennes, $\mathrm{n}^{\circ} 51$ (nouvelle série) spécial « Les religions et croyances populaires au Vietnam », Hanoi, 1996, pp. 80-93.

\section{NOTES}

1. Le vocabulaire français ne permettant pas de rendre avec exactitude le sens des termes viêtnamiens, j'ai opté pour la conservation des termes viêtnamiens. Toutefois, le lecteur trouvera dans le texte et les notes de bas de page des remarques qui lui permettront de mieux cerner les idées que ces mots recouvrent.

2. Thánh Mẫu : le premier terme de cette expression évoque la pureté, la "grandeur ", le sacré et le second terme renvoie à l'idée de maternité. L'idée générale est donc celle d'une "Mère Sacrée ", « Vénérable Mère » voire "Sainte Mère ».

3. Pierre Jean SIMON, Ida SIMON-BAROUH, Hâù bóng, un culte viêtnamien de possession transplanté en France, Cahiers de l'Homme, 1973.

4. On note actuellement au Viêt Nam un très fort engouement pour ce culte, les cérémonies autorisées rassemblent des milliers de personnes venues de différentes régions du pays ou de l'étranger.

5. Pour l'enquête, depuis 1990, j'ai suivi les adeptes dans différentes régions de France: Aquitaine, Bretagne, Bourbonnais, et banlieue parisienne. Nous confronterons les pratiques actuelles avec celles décrites en 1967, à Ste Livrade, et celles du Viêt Nam, lieu de référence, où en 1995 et 1996, j'ai assisté à des cérémonies, rencontré des médiums à Ho Chi Minh ville (Saïgon), Hà Noi et Huê.

6. "thần" terme évoquant le merveilleux, a le plus souvent été traduit par "génie " par les différents auteurs en français.

7. Les hommes médiums sont appelés : ông dồng, (monsieur serviteur).

8. Cette organisation du culte à partir de "Quatre Palais » n'est pas la seule existant au Viêt Nam. Il existe également une variante dite des «Trois Palais» reposant essentiellement sur les dimensions céleste, aquatique et celle des monts et forêts. Le registre terrestre n'y occupe pas le même rang que dans le culte que nous avons choisi d'étudier.

9. Si la forme actuelle du culte est largement inspirée par l'organisation sociale du XIX ${ }^{\mathrm{e}}$ siècle on trouve des traces de ce culte dès le XVIII ${ }^{\mathrm{e}}$ siècle (information non publiée de Nguyen Tùng).

10. Le panthéon semble constitué de différents avatars. Les Thánh Mẫu sont les trois aspects les plus élevés d'une grande déesse-mère primordiale (Mẫu), mère de toute vie, les dames et suivantes sont leurs avatars. Les mandarins sont eux les fils des rois dragons, grandes figures de la mythologie viêtnamienne et les princes en sont à leur tour les avatars. 
11. Terminologie empruntée à Luc DE HEUSCH in Pourquoi l'épouser ? et autres essais, qui qualifie ainsi les possessions acceptées où l'esprit est perçu comme un bien, en opposition aux possessions dites « malheureuses » où l'esprit est un mal qu'il faut exorciser.

12. Notons toutefois une exception, le général Trần Hýng Đạodont les incarnations se passent de façon plus violente, mais ceci est le fait d'une minorité parmi les médiums qui forment un groupe à part dans le culte, car ils ne sont possédés que par ce seul génie.

13. Ceci vaut pour le culte pratiqué au Nord. À Huế, les possessions collectives semblent la règle ; j'ai recensé ainsi au cours d'une cérémonie, des séquences de quinze médiums incarnant simultanément des thánh.

14. Les phases sont successivement : 1 - la descente du thánh (la tête et les épaules du médium sous le voile rouge de la possession). 2 - son habillage par les assistantes. 3 - prosternations et offrande d'encens. 4- "danse" du thánh conçue comme son "travail» (làm viêc). 5consommation de boissons, cigarettes ou chiques de bétel avec éventuellement audition de musique et de requêtes, et réponses. 6 - distribution du «bonheur » (lọc) sous la forme de fruits, légumes, etc...7 - départ du thánh (à nouveau sous le voile rouge)

15. « đồng " :étymologiquement, ce mot désigne, en chinois, un jeune garçon esclave et soumis de ce fait au célibat. Le célibat impliquant la virginité, la pureté, état sine qua non pour réaliser la possession; cf. DURAND, 1959, p. 10.

16. " giá » : l'expression complète " giá lâm » signifie l'arrivée impériale. L'idée ici est celle d'un « retour » dans le corps (du médium). En viêtnamien, le mot giá exprime l'idée de prix, de valeur ; les médiums utilisent très fréquemment ce terme giá pour évoquer l'incarnation d'une entité.

17. À partir du constat de l'existence de cultes masculins et féminins, il serait intéressant d'approfondir la question de la distinction entre possession masculine et féminine au Viêt Nam, de s'interroger notamment sur la nature de la relation entre médium et esprit, selon le sexe.

18. La conversation s'effectue en viêtnamien, le langage du génie étant stéréotypé est parfois difficile à comprendre pour les néophytes. De plus le thánh s'adresse rarement à la cantonade ; la plupart du temps, les propos sont tenus soit à l'une des assistantes qui les transmet à la personne concernée, soit directement à la personne mais de façon confidentielle. Dans ce cas précis, la femme, sans être bà đồng était une assistante assidue de la bà đồng qui officiait ce jour-là. C'est en discutant ensuite avec cette personne que j'ai su ce qui s'était dit.

19. Tous les adeptes se revendiquent bouddhistes en premier lieu et ne voient pas de contradictions à associer les thánh et le Bouddha qui, par ailleurs, est unanimement reconnu comme la divinité suprême.

20. «carrière » fait partie le plus souvent du vocabulaire de l'entreprise professionnelle mais les anthropologues sociaux et les sociologues l'ont repris en lui octroyant une dimension plus large. Nous citerons ici Erwin GOFFMAN pour l'expliciter : « il (le concept de carrière) est employé dans une acception plus large, pour qualifier le contexte social dans lequel se déroule la vie de tout individu. On se place alors dans la perspective de l'histoire naturelle, c'est-à-dire que l'on néglige les simples événements pour s'attacher aux modifications durables, assez importantes pour être considérées comme fondamentales et communes à tous les membres d'une catégorie sociale, même si elles affectent séparément chacun d'entre eux. » Ainsi : « l'intérêt du concept de carrière réside dans son ambiguité. D'un côté, il s'applique à des significations que chacun entretient précieusement et secrètement, image de soi et sentiment de sa propre identité ; de l'autre, il se réfère à la situation officielle de l'individu, à ses relations de droit, à son genre de vie et entre ainsi dans le cadre des relations sociales. Le concept de carrière autorise un mouvement de va et vient du privé au public, du moi à son environnement social, qui dispense de recourir abusivement aux déclarations de l'individu sur lui-même ou sur l'idée qu'il se fait de son personnage ». GOFFMAN, 1968, p. 179.

21. Les traits choisis ne sont pas singuliers au culte étudié et l'on pourrait recourir aux mêmes éléments pour étudier les conditions d'autres pratiques cultuelles. 
22. À ne pas confondre avec chùa, la pagode réservée exclusivement au culte bouddhique.

23. La construction de la pagode a commencé en 1983 sur un terrain de la commune donné à l'association bouddhiste. Ouverte depuis plusieurs années, la pagode n'a été inaugurée officiellement qu'en juillet 1997.

24. Notons que le rideau séparant le religieux du profane est un usage actuel très courant au Viêt Nam pour l'autel des Ancêtres que l'on trouve en bonne place dans la pièce principale de la plupart des maisons.

25. ROUGET, 1990.

26. Nous reprenons ici la terminologie employée par Maurice Durand pour désigner les textes liturgiques chantés au cours des cérémonies. Le mot viêtnamien est : «chầu văn ", et d'après cet auteur, in op.cit., p. 34 : «Les chầu văn sont (donc) des compositions littéraires que nous traduirons par "hymnes" qu'on récite pour rendre hommage aux génies considérés comme des rois, des princes ».

27. Communication personnelle de Nguyến Tùng, CNRS-LASEMA.

28. La danse étant comprise comme un «travail», le devoir qu'il doit accomplir lors des incarnations.

29. Sur ces deux formes d'acculturation, voir le chapitre II des conclusions, «les deux

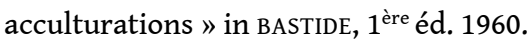

30. Contrairement à d'autres entités attachées au territoire d'origine, comme les génies tutélaires des villages par exemple, les thánh s'incarnent dans les médiums sans tenir compte du pays de résidence de ces derniers.

\section{RÉSUMÉS}

Au-delà de la description, en France, du culte de possession viêtnamien dit des Thánh Mẫu («Saintes » Mères) ou des thánh tứ phủ («génies» des Quatre Palais), cet article tente de comprendre comment, en situation migratoire, cette pratique religieuse se perpétue tout en se transformant. Les nouvelles conditions de vie, d'une part, qu'elles soient matérielles ou culturelles, entraînent une modification des pratiques, amenant les adeptes, mais aussi les «divinités» à s'adapter au contexte français. D'autre part, la migration tend à inscrire la population viêtnamienne dans un rapport minoritaire avec la société d'accueil. Cette situation particulière, impose, de façon explicite ou non, des contraintes, des restrictions sur les possibilités d'expressions culturelles des minoritaires, ce qui a modifié, certains aspects du culte, tant au plan des pratiques, que des fonctions elles-mêmes. Ainsi, les implications sociales de cette pratique religieuse sont, en France, plus restreintes, au regard, à la fois de la taille du groupe concerné, et de la société globale; et, fonction nouvelle, le culte participe de plus en plus à l'expression d'un sentiment d'appartenance ethnique.

Beyond the description of such Vietnamese possession cult, in France, as Thánh Mẫu ("Holy Mothers") or thánh tứ phủ ("genies" of the Four Palaces), this article strives to understand how, in a migratory situation, this religious practice can last, while undergoing changes. On the one hand, new living conditions, whether of a material or a cultural kind, lead to alterations in religious practices, pushing followers, but also "deities," to adjust to the French context. On the other hand, migration tends to put Vietnamese people in a minority position to their host society. The particular situation of minorities generates - either implicitly or not - constraints 
and limitations in the range of their cultural expression, and it has altered certain aspects of the rites, not only within the practice itself, but as far as its very purpose is concerned. Thus are social implications of such religious practices more restrained in France, both as to the size of the concerned group, and as to society as a whole; and, what is a novelty, rites play an everincreasing function in the expression of feelings of ethnic belonging.

Más allá de la descripción, en Francia, del culto de posesión vietnamita conocido commo Thánh Mẫu ("Santas" Madres) o thánh tứ phủ ("genios" de los Cuatro Palacios), este articulo trata de compreder como, en situación migratoria, esta práctica religiosa se perpetua al mismo tiempo que se transforma. Las nueves condiciones de vida por un lado, sean materiales o culturales, implican modificaciones de las prácticas llevando a los adeptos pero también a las "divinidades » a adaptarse al contexto francés. Por otra parte, la migración tiende a incribir la población vietnamita en una relación minoritaria con la sociedad de acogida. Esta situación particular impone, de manera explicita o no, dificultades y restricciones sobre las posibilidades de expresiones culturales de los minoritarios, lo cual ha modificado algunos aspectos del culto tanto en el terreno de las practicas como en las mismas founciones. Asi pues, las implicaciones sociales de esta práctica religiosa son. en Francia, más restringidas en relación a la vez con el tamaño del grupo en cuestión y de la sociedad global ; y, función nueva, el culto participa cada vez más en la expresión del sentimiento de pertenencia étnica.

\section{AUTEUR}

MARTINE WADBLED

LASEMA-CNRS Paris, CERIEM-UHB Rennes 